\title{
¿Las redes virtuales de aprendizaje son mediadoras en la construcción de conocimiento escolar?, revisión de algunos antecedentes de investigación
}

Are the virtual learning networks as mediators in the construcction of school knowledge? a review from some background research

Ruth Molina Vásquez ${ }^{1}$

\begin{abstract}
Resumen
A partir de la reflexión sobre la forma como las redes virtuales de aprendizaje (RVA), cualifican procesos de aprendizaje en la escuela, se presentan algunos elementos centrales de proyectos de investigación que se han realizado en relación al tema. Se utiliza la metodología de análisis documental para categorizar los problemas que orientan estas investigaciones, sus objetivos, metodologías, elementos teóricos y conclusiones. A partir de lo anterior, se establece una breve reflexión sobre las posibilidades mediadoras que ofrecen estas RVA en los procesos de construcción de conocimiento escolar.
\end{abstract}

\section{Palabras clave:}

Redes virtuales de aprendizaje, conocimiento escolar, aprendizaje, antecedentes de investigación.

\begin{abstract}
From reflection about how the virtual networks learning-RVA-, qualify processes of learning in the school, this paper presents some elements of research projects, that have inquired about this topic. It uses the methodology of documentary analysis, to categorize the issues that guide these researchs, its objectives, methodologies, theories and conclusions. From these researchs, this papers presents a brief reflection about the possibilities by these these networks as mediators offer in the processes of construction of school knowledge.
\end{abstract}

\section{Keywords:}

Virtual networks of learning, school knowledge, learning, research background.

Docente de planta de la Facultad de Educación de la Universidad Distrital Francisco José de Caldas y estudiante del Doctorado Interinstitucional de Educación en la misma universidad. Avenida Ciudad de Quito N. 64-81 Oficina 705. Conmutador 3238400, extensión 6356-6357. Correo electrónico: rmolinav@udistrital.edu.co 


\section{Reflexión inicial}

El reto de pensar en nuevas formas de mediación que faciliten los procesos de aprendizaje de los estudiantes ha sido una preocupación constante por parte de los maestros, aún más en la actualidad, cuando en este momento histórico los avances de las tecnologías de la información y las comunicaciones ponen nuevos artefactos tecnológicos al servicio de los procesos didácticos. La exploración de estas mediaciones, en particular de las redes virtuales de aprendizaje como facilitadoras de la construcción del conocimiento escolar, es el objeto de este artículo.

Inicialmente, se realiza una revisión de investigaciones referidas a la forma como se construye este tipo de conocimiento en particular y, posteriormente, las características de las redes virtuales de aprendizaje para tratar de establecer las posibilidades que, desde los procesos de investigación referidos, tienen estas redes en los procesos de construcción de conocimiento escolar en red.

A partir de esta revisión se encuentran caminos investigativos que han sido explorados y otros que surgen como posibilidades de trabajo, que podrían aportar elementos conceptuales e investigativos para el uso de las redes virtuales de aprendizaje como mediadoras de conocimiento en la escuela.

\section{¿Cómo es el proceso de construcción de conocimiento escolar?}

El área de conocimiento escolar se configura como una línea de investigación relativamente reciente (Porlán, 1998), que ha tenido un desarrollo significativo en el área de enseñanza de las ciencias (Rodrigo, 1993; Rodrigo, 1994; García, 1998 y Martínez, 2006) y hasta el momento no reporta proyectos de investigación que se ocupen del uso de mediadores tecnológicos particulares como son las redes virtuales de aprendizaje, pero que pueden mostrar caminos interesantes para su uso.

En el área de enseñanza de las ciencias se encuentran investigaciones que se configuran como referentes para el proceso de construcción de conocimiento escolar. En estos proyectos las preguntas de investigación plateadas giran en torno de los contenidos escolares como un problema en la construcción del conocimiento escolar (Martínez, 2006), sobre su relación con la práctica profesional del docente a partir de sus intervenciones en el aula, sus procesos reflexivos y los obstáculos que dificultan la integración entre reflexión y práctica de aula (Vásquez, et al, 2006) y sobre las teorías y los modelos educativos que están a la base de la aplicación de las Tic en la educación, desde una mirada de construcción colaborativa de conocimiento (Sánchez, 2009).

FIGURA 1. Temáticas de investigación consultadas en el tema de Conocimiento escolar

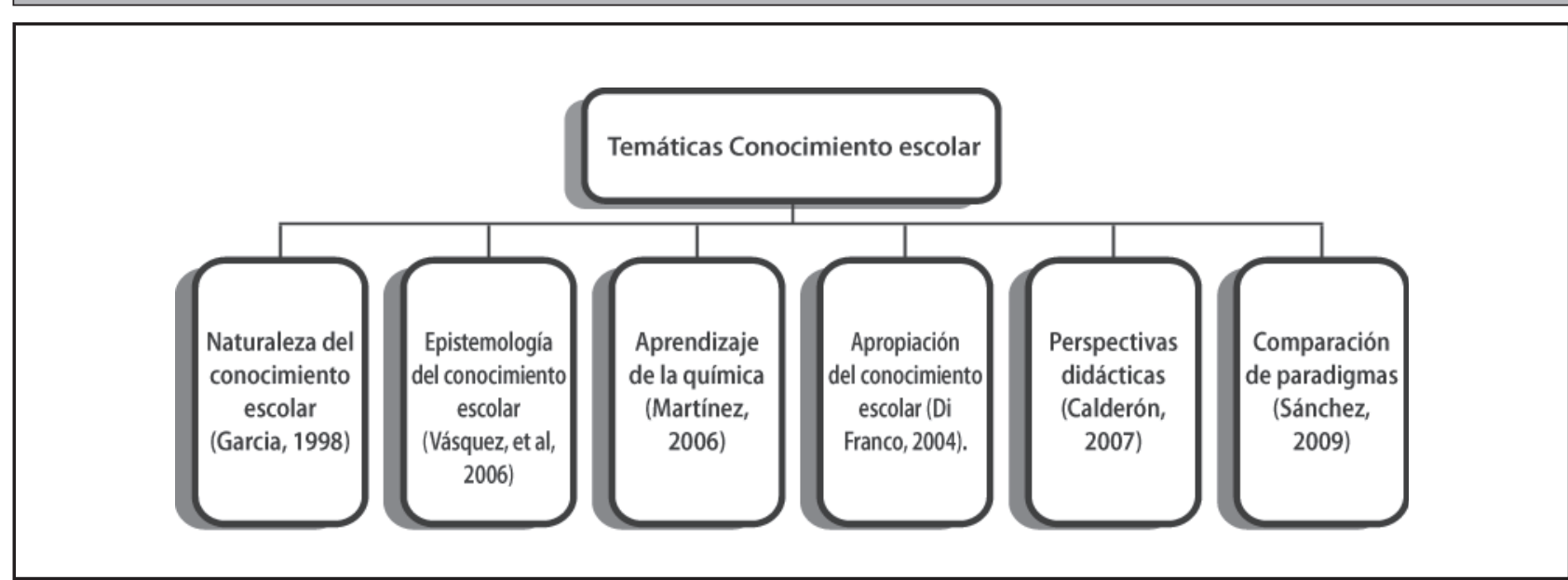

Fuente: elaboración propia

REVISTA CIENTÍFICA / ENERO -DICIEMBRE DE 2009 / No. 11 / BOGOTÁ, D.C. 
A partir de esas preguntas, los proyectos se plantean objetivos relacionados con la compresión del conocimiento profesional, desde el conocimiento escolar en la enseñanza de la química, de los procesos de apropiación de conocimiento, desde una mirada epistemológica y política, y así caracterizar y analizar críticamente las perspectivas epistemológica y didáctica del conocimiento escolar subyacente en los lineamientos curriculares y los textos de ciencias sociales (Calderón, 2007), con el fin de analizar las condiciones para crear comunidades de construcción de conocimiento (Sánchez, 2009). Aunque no es evidente que algunos de estos objetivos se alcancen dada la amplitud de su planteamiento, sí es claro que se alcanzan parcialmente o dan pistas claras para su consecución posterior.

Desde la perspectiva metodológica, estos proyectos se apoyan en investigación de carácter cualitativo, aplicado, transversal-longitudinal, descriptiva, explicativa y de campo. Bajo esta mirada, se utilizan estrategias de estudio de caso (Martínez, 2006; Calderón, 2007), observación de clase, entrevistas y reflexión. La información recolectada es objeto de análisis de contenido y análisis de la unidad didáctica, bajo la mirada de las categorías previas anotadas por los autores, tales como: el conocimiento escolar, los criterios de selección de información, la utilidad del conocimiento escolar y su construcción (Vásquez, Jiménez, Mellado y Tabeada, 2006); ejes dinamizadores, obstáculo y cuestionamiento (Martínez, 2006); y tipos de contenidos, fuentes y criterios en la elaboración de contenidos (Di Franco, Di Franco y Sideral, 2004).

Estos proyectos de investigación concluyen que es necesario profundizar en el estatus epistemológico del conocimiento escolar en la formación profesional del profesor, seleccionar fuentes sociales de recolección de información y la participación en grupos de trabajo como catalizador de nuevas teorías prácticas más allá de las tareas escolares. Esto supone un proceso de transición del conocimiento profesional del profesor respecto al conocimiento escolar, para hacerlo evidente (Martínez, 2006).

Respecto a los contenidos, el análisis se centra en las categorías de contenidos de enseñanza, en as- pectos como: temas específicos, datos y hechos, la relación entre tiempo y contenido, los límites fuertes y la relación cerrada en el dinámica de la clase, la actitud del docente frente a los contenidos, las fuentes de información utilizadas, las actividades de enseñanza-aprendizaje (Di Franco, Di Franco y Sideral, 2004).

Estos proyectos de investigación presentan elementos comunes en torno a consideraciones generales entre las cuales se destacan las siguientes:

- Se indica como referente la construcción del conocimiento escolar a partir del conocimiento profesional del profesor. Este es un elemento común, pues se plantean relaciones entre el conocimiento profesional del profesor y la manera como este influye en la construcción de conocimiento escolar o se devela el conocimiento escolar a partir del análisis de la actuación del profesor en los procesos didácticos la interior del aula de clase. En este sentido, el proyecto de investigación analizado en este artículo, centra el estudio en los procesos de interacción de los estudiantes, sin la influencia determinante del maestro, con el objeto de caracterizar el proceso de construcción de conocimiento escolar sin la variable interviniente del docente.

- Metodología de estudio de caso y observación de clase: se acude metodológicamente a una estrategia basada en el estudio de caso en la observación de lo que ocurre en el aula de clase, para recolectar la información que posteriormente es analizada de forma cualitativa.

- El conocimiento profesional del profesor es complejo, pues es un aspecto en el que influye los procesos de construcción de conocimiento escolar y el contexto de enseñanza.

- Epistemología particular del conocimiento escolar. La naturaleza del conocimiento escolar es diferente del conocimiento científico o del cotidiano, por lo cual posee características diferenciadoras.

- En los resultados se indica que el conocimiento escolar está centrado en muchas ocasiones 
en la información, en los datos y no en los conceptos. Esto implica la existencia de una fragmentación del saber en parcelas que no necesariamente están relacionadas, como el vaciamiento en palabras sin significado y la sobre información.

Un aspecto relevante de esta investigaciones es que una de ellas aporta categorías de análisis de la información centradas en ejes dinamizadores, obstáculo y cuestionamiento -ejes DOC- los cuales facilitan la comprensión del conocimiento profesional de los profesores (Martínez, 2006). Se entienden los ejes obstáculo como concepciones que posibilitan la acción, asentadas al contexto, pero que impiden el desarrollo profesional del sujeto; los ejes cuestionamiento los consideran como concepciones alternativas que sugieren otra manera de actuar pero que no se usan, por entrar en conflicto con el contexto o no considerarse funcionales, y los ejes dinamizadores, como conceptos que posibilitan el desarrollo profesional del sujeto (Martínez, 2000 y Ballenilla, 2003, citado por Martínez, 2006).

Por otra parte, se resalta la concepción de la enseñanza desde una perspectiva epistemológica (Bruner 1969 y Pruzzo 1999) y del aprendizaje como una construcción de conocimientos, siguiendo pautas similares a la producción del conocimiento científico, aunque destaca un estudio en donde el eje de esta producción es la construcción colaborativa (Sánchez, 2009). De esta manera, "el aprendizaje de las categorías constituye uno de los medios principales de socialización de los miembros en formación de una sociedad, ya que las categorías que se aprendan y utilizan habitualmente reflejan las demandas de la cultura en cuyo seno se elaboran" (Di Franco, Di Franco y Sideral, 2004, p. 222).

Conceptualmente, la mayoría de los proyectos de investigación analizados se fundamentan en aspectos basados en el desarrollo profesional del profesor, entendido como la conjugación de elementos pedagógicos, didácticos y prácticos, ligados a aspectos personales y sociales. Su relación con el conocimiento escolar es clara desde la perspectiva de Porlán cuando afirma que el conocimiento profesional del profesor:
(...) es un conocimiento sobre la integración y transformación de saberes en la perspectiva de formular determinado conocimiento escolar y sobre los procesos que facilitan su construcción (Porlan, et al, 1997, p. 161).

Así, se concibe que el conocimiento escolar es elaborado en la escuela y trasciende las explicaciones cotidianas que se desarrollan fuera de los contextos académicos, de modo que se asume como un conocimiento particular. Es una reelaboración del conocimiento científico ajustada a las características del contexto escolar, con epistemologías diferentes, actores particulares, maneras de producción y validación diferentes. Así, el análisis de una epistemología cotidiana y científica (Rodrigo, 1994) muestra que:

- El conocimiento cotidiano trabaja sobre el plano real, describe fenómenos reales, resuelve problemas prácticos, busca un conocimiento útil, sigue procedimientos heurísticos para interactuar eficazmente con el entorno, sigue un proceso de aprendizaje espontáneo y produce teorías implícitas.

- El conocimiento científico trabaja en un plano de fenómenos científicos, resuelven problemas a largo plazo, busca que su conocimiento sea cierto, aplica sus ideas al mundo idealizado de la ciencia, usa como criterio de validación de teorías su eficacia para interpretar el entorno y planificar su comportamiento, siguiendo procesos sistemáticos y exhaustivos, genera aprendizajes de forma planificada y gradual, produce teorías explícitas.

Aunque algunas corrientes sostienen que la construcción del conocimiento escolar supone un proceso de cambio del conocimiento cotidiano al conocimiento científico, este cambio conceptual es una tarea difícil, puesto que el conocimiento cotidiano es particularmente resistente al cambio (Rodrigo, 1993). De otra parte, la hipótesis de continuidad entre conocimiento escolar y científico reducen, al primero, a una versión empobrecida del segundo, aunque claramente se diferencian por el entorno físico, actores, metas, intenciones, actividades, discursos, entre otros (Rodrigo, 1994). 
$\mathrm{Al}$ interior del presente trabajo, se considera que, de ello deriva que la escuela debe proporcionar entornos para el aprendizaje contextualizado de los tipos de conocimiento que se integran, pues están alimentados por diferentes epistemologías, y el estudiante debe diferenciarlos en función de contextos y usos diversos.

La construcción del conocimiento escolar es entendida como la complejización del conocimiento cotidiano referido a los problemas del mesocosmos, es decir, del mundo cercano a los alumnos, con miras a hacer un acercamiento a los problemas del macrocosmos y el microcosmos, que corresponden a la esfera del conocimiento científico. La idea no es cambiar un tipo de conocimiento por otro, sino realizar una integración didáctica de estos dos y de otras formas de conocimiento (Valbuena, 2007), ya que estas diferentes formas de conocimiento no se oponen, sino que existe una continuidad entre ellas (García, 1998).

Esto permite al conocimiento escolar, organizar permanentemente el conocimiento, jerarquizarlo y presentarlo en un sistema procesual que se revela en los currículos escolares. Sin embargo, tal como lo manifiesta la investigación de Martínez (2000) y Van Driel, et al.,(2002), la práctica profesional de los profesores en ciencias, no siempre permite establecer las fuentes y selección de contenidos, validez y los referentes utilizados en esta perspectiva, como ejes dinamizadores, Incluso demuestran que los profesores no tienen un conocimiento muy profundo acerca de las dificultades de aprendizaje de los estudiantes.

El conocimiento escolar es un proceso constructivo, evolutivo, propio de la escuela, que aborda elementos relevantes del contexto (Martínez, 2000), que se fundamenta en complejos elementos epistemológicos y constructivistas, orientados a la evolución, construcción y negociación de conocimiento, el aprendizaje significativo y la interacción social; e igualmente, ideológicos-críticos, fundamentados en el enriquecimiento del conocimiento mediante la comunicación y la cooperación (García, 1998).
Esta perspectiva se contrapone a aquellos procesos de cambio conceptual que consideran al conocimiento escolar como equivocado o inferior, y a sus construcciones como concepciones erróneas, ideas equivocadas, creencias, supersticiones o preconceptos (Cubero, 1994), por lo cual debe ser "reemplazado" por el conocimiento científico entendido como un conocimiento "verdadero".

Es necesario realizar un proceso de reconocimiento de las concepciones alternativas de los estudiantes, como un paso inicial de la construcción del conocimiento escolar, a partir del cual se acude a un proceso de negociación de significados, de interacción social con otros y del uso de mediadores, en este caso tecnológicos, adoptando una postura de carácter constructivista.

Esto implica que la construcción de conocimiento es un proceso gradual, dinámico y evolutivo, el cual se debe evidenciar en investigaciones, para favorecer esa construcción. Estos referentes dan pie a la formulación de una progresión que da cuenta de los procesos realizados, cambios en los sistemas de ideas, progresión conceptual, procedimental, actitudinal y procesos de interacción en la construcción de conocimiento escolar (García, 1998).

La organización del conocimiento escolar requiere de tramas conceptuales y jerarquización del contenido, mediante tres formas de organización (García, 1998) que corresponden a un primer nivel de conceptos metadisciplinares entendidos, como lo señala García, como integradores que se basan en la complementariedad de términos opuestos, que se originan en la búsqueda de lo común de los sistemas físicos, biológicos y sociales, son productos culturales no categorías lógicas generales y se constituyen en un marco de referencia para la construcción del conocimiento escolar.

Un segundo nivel comprende la red de interacciones, tramas de contenido y campos conceptuales que permiten concretar didácticamente las relaciones entre las ideas, en forma de contenidos de referencia y la explicitación de sus relaciones de jerarquía por medio de diagramas de representación, como los mapas conceptuales. El tercer nivel 
de organización considera los tópicos de contenido como campos conceptuales integrados que permiten reconocer unos límites claros, estableciendo así unos elementos didácticos favorables para la comprensión de los contenidos.

Esta organización se evidencia en la formulación de hipótesis de progresión, entendida como niveles para la organización dinámica del conocimiento escolar, mediante propuestas de transición de unos niveles a otros. De esta manera, se evidencia la organización del conocimiento escolar de forma jerarquizada, procesual, relativo, como un sistema de ideas que se organiza continuamente en interacción con otros sistemas y que se concreta curricularmente en una hipótesis de progresión, que se refiere tanto a un contenido concreto, como a un conjunto de contenidos conectados en tramas (García, 1994).

En este punto, es necesario poner en consideración que, de acuerdo con Vyggotski (1934), en los niños los conceptos se forman a partir de las interacciones verbales con los adultos que, en el contexto escolar, orienta deliberada y explícitamente la adquisición de conocimientos sistematizados, elabora significados y explicita relaciones lógicas. Esta interacción se entiende como una relación comunicativa, en donde la palabra es medio para analizar y destacar atributos, abstraerlos y sintetizarlos. Los conceptos son productos históricos y significantes de la actividad mental y social, que, para su formación, pasan por al menos tres etapas: conglomerados, colecciones y pseudoconceptos.

Si bien se considera que estos procesos tienen una fase de construcción individual, también se debe reconocer que los conceptos son representaciones colectivas, que agregan a la experiencia individual la sabiduría acumulada por la sociedad a lo largo de los siglos (Durkheim, 1982, citado por Rodrigo, Rodríguez y Marrero, 1993). Se reconoce así la importancia de la interacción social en la formación de las representaciones colectivas y del conocimiento como fenómeno social que trasciende las dinámicas individuales, pues aunque se evidencie en él, este no las construye en solitario, ya que forma parte de grupos y clases sociales.
La interacción es fundamental en la construcción colaborativa de conocimiento y desde lo pedagógico se entiende como un sistema de interacciones cuidadosamente diseñado que organiza e induce la influencia recíproca entre los integrantes de un equipo en un proceso que se desarrolla gradualmente, en el que todos son mutuamente responsables del aprendizaje de los demás (Johnson y Johnson, 1998).

Todos los elementos citados anteriormente, apuntan a concebir el conocimiento escolar como resultado de la interacción entre el conocimiento cotidiano y el conocimiento científico, mediante un proceso de construcción que se realiza de forma dinámica, flexible, gradual, que muestra la evolución realizada por los estudiantes y en la que intervienen distintos mediadores que favorecen esa construcción. Este proceso se evidencia mediante unos momentos de transición o progresión de la construcción conceptual y que dan cuenta de la manera como los sistemas de ideas de los estudiantes cambian, se transforman.

A partir de estos elementos, es posible diseñar un ambiente didácticamente enriquecido y orientado a facilitar esas interacciones entre un grupo social, de tal manera que a partir de ellas se puedan elaborar representaciones colectivas que tomen forma de un concepto particular.

De otra parte, es interesante la reflexión sobre la organización del conocimiento escolar mediante hipótesis de progresión, que permite vislumbrar los niveles de construcción conceptual, además de considerar las características procesuales, dinámicas y jerarquizadas. En este punto, la reflexión se orienta a revisar cuáles estrategias y recursos pueden convertirse en mediadores para facilitar estos niveles de construcción del conocimiento que se construye en la escuela, de tal manera que sea un proceso gradual, dinámico y colaborativo.

Las anteriores, son algunos de los aspectos que caracterizan el apoyo que brindan las tecnologías de la información y la comunicación a los procesos de enseñanza y aprendizaje escolar en red. Dentro de ese amplio panorama de posibilidades y tipos de 
tecnologías que se pueden utilizar, se encuentran las redes virtuales de aprendizaje, que podrían ser objeto de mediación para la construcción de conocimiento escolar.

\section{¿Las redes virtuales de aprendizaje facilitan la construcción de conocimiento escolar?}

El campo de investigación en redes virtuales de aprendizaje es relativamente reciente, por lo cual se encuentran escasos proyectos de investigación en torno a esta temática. Una de estas investigaciones es la de Inter Cutural Learning Network, que generó un proyecto que usó el correo electrónico para conectar a estudiantes de educación secundaria de San Diego California, con otros escolares de Alaska, Japón, México, Puerto Rico e Israel, realizado por Cohen y Riel en el año 1989 (Harasim, 2000).

En la formación universitaria se han realizado experiencias de investigación comparando el grado de interactividad entre los estudiantes que participan en cursos en red y aquellos que lo hacían de forma presencial en la Simon Fraser University, donde los estudiantes que utilizaron la red presentaron mayor número significativo de interacciones en comparación a quienes estuvieron de forma presencial (Harasim, 2000), esto debido a que presentan mayor interacción con el profesor, comodidad y más fácil acceso a la ayuda necesaria, generación de aprendizaje colectivo y aumento de la motivación. En esta misma línea, el New Jersey Institute of Tecnology construye una plataforma de aula virtual y encuentra un mayor impacto, aceptación y motivación de los estudiantes hacia los cursos en red (Harasim, 2000).

Garrido (2003) realiza un estudio etnográfico de la comunidad virtual www.gc-red.com, con el fin de identificar cómo las comunidades virtuales sirven de contexto para el aprendizaje y la generación social de conocimiento. El objetivo de esta investigación es profundizar en el conocimiento de las comunidades virtuales desde la perspectiva de la teoría del aprendizaje social, a partir de un marco teórico y de análisis para estudiar las características de las comunidades de práctica (Wenger, 2001) en el contexto de comunidades sociales en la virtualidad. Las categorías de análisis propuestas son: el contexto, a través de las dimensiones de la práctica que dan coherencia a la comunidad en un espacio virtual, y la identidad de participación de los usuarios en sus interacciones y en la negociación de significados. Metodológicamente se realiza una interpretación desde una perspectiva etnográfica.

Este proyecto concluye que la construcción de una identidad de participación mediante la experiencia individual y social de desarrollar relaciones de interacción activa en la negociación de significados, desde la perspectiva de la afiliación por el compromiso a comunidades virtuales, posibilita el aprendizaje de un conocimiento generado socialmente.

En la misma temática de comunidades virtuales, el "Estudio de la estructura y del comportamiento de las comunidades virtuales de aprendizaje no formal sobre estandarización del e-learning" (Burgos, 2006), se propone definir el concepto, las características y los estándares de la formación online o educación virtual y definir e identificar las características de las comunidades virtuales de aprendizaje no formal sobre estandarización del e-learning. La comunidad virtual es entendida como "cualquier grupo de usuarios que se establece en torno a una temática o actividad iniciales concretas y que desarrolla una comunicación fundamentalmente asíncrona, pero también síncrona, y una compartición de información, opiniones y recursos a través de una plataforma o entorno online" (Burgos, 2006:120)

Mediante un estudio comparativo de tres comunidades, concluye que estas están compuestas por nodos de información representadas por sus miembros y las relaciones entre ellos, que los mecanismos de participación mediante recompensa así como los de altruismo, son los dos principales acicates en la contribución individual a la comunidad. De igual manera, se comprueba que los encuentros presenciales son especialmente útiles para la riqueza e incentivación de la comunidad virtual. Este es un aspecto importante de reflexión 
en el presente trabajo, sin embargo, la presencialidad puede ser considerada como una variable que interviene en el estudio y puede influir en el proceso de construcción de conocimiento escolar, por lo cual se considera que no es conveniente incluirla en la estrategia metodológica de trabajo.

A pesar de no configurarse dentro de un proyecto de investigación formal, se encuentran a nivel internacional experiencias de maestros como la del Equipo LaceNet, ${ }^{2}$ una asociación de profesores de Catalunya interesados por el uso educativo de las Tic. Desde el año 1995, ofrece proyectos telemáticos en los centros educativos, los cuales son diseñados para una etapa educativa concreta, están integrados en el currículo escolar habitual y han ido diversificando la oferta y ampliando el margen de edad de los niños y niñas a los que van destinados.

Estos proyectos se basan en el trabajo cooperativo y en la comunicación entre los diferentes grupos que participan, son interdisciplinarios y se plantean con suficiente flexibilidad para que cualquier centro pueda seguirlos, a partir de unas propuestas mínimas muy sencillas y una serie de actividades complementarias para aquellos grupos que quieran profundizar más. Actualmente, se cuenta con proyectos dirigidos al nivel de educación infantil, escuela primaria y los dos primeros cursos de ESO.

Con esta misma orientación de trabajo por proyectos se encuentra en $\mathrm{iEARN}^{3}$ (International Education and Resource Network), una organización no lucrativa compuesta por más de 30.000 escuelas y organizaciones juveniles en más de 130 países, que invita iEARN faculta a los profesores y los jóvenes a trabajar juntos en línea usando el Internet y otras nuevas tecnologías de comunicación. a profesores y jóvenes a trabajar juntos en línea usando Internet y otras tecnologías de comunicación. Más de 2.000.000 estudiantes cada día se dedican a la colaboración de todo el mundo proyecto de trabajo. Desde 1988, iEARN ha sido pionera en los vínculos entre la escuela en línea para que los estudiantes puedan participar en proyectos educativos

\footnotetext{
2 Disponible en: http://www.lacenet.org

3 Disponible en: http://www.iearn.org
}

REVISTA CIENTÍFICA / ENERO -DICIEMBRE DE 2009 / No. 11 / BOGOTÁ, D.C. significativos con sus compañeros en sus países y en todo el mundo.iEARN ha sido pionera, estableciendo vínculos entre las escuelas en línea para que los estudiantes participen en proyectos educativos significativos con compañeros de otros países.

iEARN es: iEARN está dirigida a una comunidad incluyente y culturalmente diversa, un entorno seguro y estructurado en el que los jóvenes pueden comunicarse proporcionando un entorno seguro y estructurado, en el cual los jóvenes pueden comunicarse una oportunidad de aplicar los conocimientos en servicio de proyectos de aprendizaje y aplicar sus conocimientos en servicio de proyectos de aprendizaje y conformar una comunidad de educadores y alumnos hacer una diferencia, como parte del proceso educativouna comunidad de educadores y alumnos. Posee más de 150 proyectos diseñados para que los profesores y los estudiantes los adapten e integren a sus planes de estudio y las necesidades del aula y los horarios, participen en espacios de foro en línea para encontrarse con grupos de todo el mundo que estén trabajando en el mismo proyecto.

A nivel nacional no se han encontrado trabajos de investigación sobre la formulación, conformación o implementación de redes virtuales de aprendizaje orientados a la construcción de conocimiento escolar en temáticas particulares como la educación en tecnología, aunque cabe señalar que se encuentra un espectro amplio de trabajos orientados a la incorporación de las Tic en el aula.

Luego de una búsqueda inicial en diferentes bases de datos, ${ }^{4}$ se encuentran culminados sobre el tema de redes virtuales de aprendizaje, algunos proyectos de investigación que han sido avaladas por el Ministerio de Educación Nacional. A continuación, se presenta un análisis transversal de estos trabajos, a partir de los problemas de investigación planteados, los objetivos, elementos teóricos relevantes, metodologías aplicadas y resultados de investigación obtenidos.

4 Entre estas bases de datos se encuentran: ERIC (Educational Resource Information Center), ProQuest Education Journals, DialNet, Dialnet, SpringerLink, Social Sciences Citation Index (ISI), Teacher's Reference. 


\section{FIGURA 2. Investigaciones consultadas sobre redes virtuales de aprendizaje a nivel nacional}

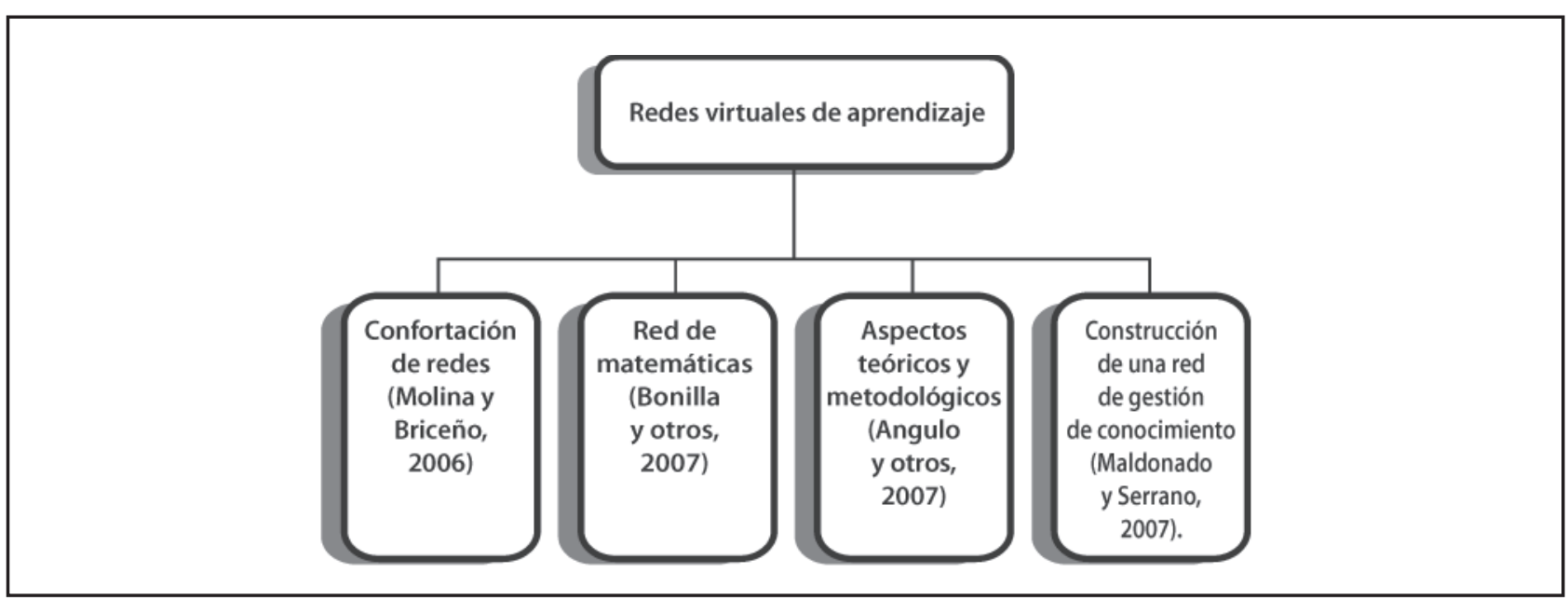

Fuente: elaboración propia

Los problemas de investigación, planteados en los proyectos, presentan como elemento común la necesidad de determinar los aspectos teóricos y metodológicos que permitirían consolidar las redes de aprendizaje las cuales se orientan, en todos los casos, a la formación de docentes. Es así como se pregunta por las características pedagógicas, comunicativas y técnicas que debe poseer un modelo de formación de docentes, en entornos virtuales, en el tema de aplicación las tecnologías de la información y la comunicación en red, (Angulo, et al., 2007), o por estas mismas características referidas directamente a la conformación de una RVA (Molina y Briceño, 2006), o cómo estos elementos se configuran en un espacio de red virtual que busca la participación colaborativa de los docentes del área de matemáticas cuando se implementa el uso de tecnologías en el aula (Bonilla, et al., 2007).

Esta orientación de los problemas de investigación muestra que la línea se encuentra en un nivel inicial de carácter descriptivo, pues apenas uno de los proyectos avanza sobre un modelo de diseño definido y orienta su problema hacia determinar el impacto de dos estrategias de participación en la consolidación de una RVA ya conformada (Molina y Briceño, 2009).

Los objetivos se enfocan hacia la conformación y consolidación de una red de aprendizaje de docentes en diversas áreas de conocimiento, a siste- matizar el modelo pedagógico propuesto para la formación de docentes mediante la estrategia de conformación de la RVA (Molina y Briceño, 2006), caracterizar estrategias de reflexión y entornos de interacción que promuevan el desarrollo de profesores a partir de la interacción en una RVA (Bonilla, et al., 2007) y determinar los aspectos teóricos y metodológicos que permitirían hablar de una red de aprendizaje consolidada (Angulo, et al., 2007)

La mayoría de los proyectos de investigación se desarrollan con una metodología de carácter integral o cualitativo, bien sea de carácter longitudinal o integrando aspectos cualitativos y cuantitativos. Se utilizan instrumentos de recolección de información como registro de protocolos automatizados de los espacios sincrónicos y asincrónicos de comunicación y colaboración en la red, entrevistas a los participantes, cuestionarios iniciales y finales que dan cuenta de la evolución de la red, cuadernos viajeros y registros de ingreso y uso de la red. Solo uno de los proyectos utiliza una metodología cuantitativa basada en la teoría de grafos, desde la cual calculan la probabilidad de que un nodo elegido al azar tenga un determinado grado de comunicación, para construir una función de distribución de probabilidad de grado.

En relación a la conformación y consolidación de las redes virtuales de aprendizaje, las conclusiones indican que estas son la representación de una 
comunidad de práctica mixta que integra actores como investigadores, docentes, usuarios del servicio o auxiliares de investigación como intermediarios y dinamizadores del proceso.

El carácter mixto de la red, es decir, que conjuga elementos virtuales y algunas actividades presenciales, marca una tendencia hacia una red de libre escala, lo que indica que es necesario cambiar el enfoque tradicional de gestión de conocimiento instruccional, para adoptar uno de aprendizaje colaborativo, creando condiciones fundamentales para la conformación de la red. El aprendizaje colaborativo es entendido como un sistema de interacciones cuidadosamente diseñado, que organiza e induce la influencia recíproca entre los integrantes de un equipo, en todos son mutuamente responsables del aprendizaje de cada uno de los demás (Johnson y Johnson, 1998, p.1), a partir del desarrollo de destrezas cooperativas para aprender y solucionar los problemas y acciones educativas en las cuales se ven inmersos (Gross, s.f.)

En cuatro casos (Molina y Briceño, 2006; Bonilla, et al., 2007; Angulo, et al., 2007; Molina y Briceño, 2009), se adopta un modelo de diseño fundamentado en elementos pedagógicos, técnicos y comunicativos que mostraron interrelaciones teóricas y metodológicas que explican las condiciones de consolidación de esta red de aprendizaje y permiten detallar aspectos metodológicos tendientes a describir, categorizar y analizar el proceso de conformación de las redes, la continuidad de la comunicación, los niveles de participación de los docentes y su interacción. De otra parte, mostraron su efectividad para promover la reflexión sobre la práctica a partir de los sistemas de instrumentos, diseñados explícitamente para su formación en el tema de implementación de Tic en el aula o el abordaje de plataformas y programas informáticos específicos en la didáctica de las matemáticas a partir de la gestión en aula, por ejemplo con el uso de calculadora electrónica.

En relación a los procesos de participación y comunicación, se encontró que las conexiones ente los participantes deben ser fortalecidas mediante actividades conjuntas que pueden ser adelantadas a través los portales utilizados para las redes y cada grupo de participantes de una institución deben constituirse como un nodo dinámico que genere nuevas aristas de contacto con otras instituciones y grupos, con la transferencia de resultados o con el ofrecimiento de servicios de extensión.

Se encuentra en uno de los proyectos (Molina y Briceño, 2006), que la sistematización del modelo pedagógico propuesto fue reflejado en el espacio virtual de forma detallada y se realizó la sistematización automatizada de los registros de interacción de los participantes, ${ }^{5}$ estos permitieron afinar y guardar de manera efectiva los datos recolectados. Este modelo se centró en un enfoque constructivista de interacción social, de comunicación multidimensional y con fundamento tecnológico abierto y flexible.

Se encuentra que los docentes participantes en las redes, las perciben como una novedad, ${ }^{6}$ la posibilidad de comunicarse con sus pares y establecer con ellos discusiones que les permiten aclarar sus puntos de vista, además de encontrar aportes que enriquecen su formación y les da la posibilidad de adaptar a su práctica los recursos que aparecen en la red.

Un aspecto común y relevante es que, en ninguna de estas investigaciones, se hace alusión a la relación de los procesos de formación de los docentes o sobre el conocimiento didáctico del profesor con la construcción de conocimiento escolar, ni siquiera de manera tangencial. Sin embargo, queda un gran interrogante: ¿el proceso de interacción de los docentes al interior de la red será equiparable al proceso de los estudiantes que interactúan en ella? Con base en los resultados de estos proyectos, se puede afirmar que es posible desarrollar una RVA que se configure como un recurso mediador potente en la construcción de conocimiento escolar dado que permite plantear actividades de aprendizaje colaborativo y guardar de forma confiable los procesos de interacción que se presentan.

5 Estos registros fueron analizados de acuerdo con las categorías de manejo de contexto académico, reconocimiento del trabajo del otro, aspectos de convivencia en red, relaciones afectivas y pertenencia a la red.

6 Esta novedad radicó en que, para la mayoría de los docentes, era la primera vez que participaban en una experiencia en RVA. 
Los proyectos de investigación consultados, presentan diversas posturas conceptuales frente al concepto de RVA, concibiéndola como:

(...) una comunidad que basada en la comunicación, crea una organización con el objeto de construir conocimiento" (Maldonado y Serrano, 2008:217)

"un tipo específico de comunidad virtual que se enfoca exclusivamente en cubrir las necesidades de aprendizaje, dentro de un contexto formal, en el marco de un espacio de encuentro regulado en la red, en el cual se puede preguntar, solucionar problemas, acceder a información, desarrollar estructuras conceptuales, realizar construcción de conocimiento, es decir, aprender sobre un tema. (Molina y Briceño, 2006, p. 22).

(...) una comunidad de personas que se disponen a trabajar en red, se caracterizan por su interés en compartir, aceptar o debatir ideas y la voluntad de aprender y de enseñar; por lo que es necesario un medio de comunicación que permita la interactividad entre los participantes, ya que de allí surgen los aprendizajes (Angulo, Garzón, Pabón, Mejía, Obando y Posada, 2007).

Estas concepciones presentan elementos comunes en relación a que la RVA se construye en un espacio virtual y está compuesta por personas que interactúan entre sí con el objeto de construir conocimiento, lo cual es coherente con algunos elementos teóricos. En primera instancia, las RVA se ubican en el ciberespacio, entendido como un punto de encuentro, sin lugar geográfico, en donde se crean nuevas relaciones de acuerdo con intereses comunes de los individuos que interactúan en él, en una compleja red social, configurando estilos de vida propios de una cibercultura (Levy, 1997).

En ese entendido, una red de aprendizaje remite a la noción de un espacio compartido por un conjunto de individuos, en el que se propicia el aprendizaje conjunto a través del uso de herramientas e instrumentos que potencian la interacción entre estos (Ochoa y Petrizzo, s.f.), a partir del intercambio de experiencias de trabajo en conjunto y de construcción de conocimiento, por tanto:

(...) son grupos de personas que usan redes de comunicación en entornos informáticos, para aprender de forma conjunta en el lugar, el momento y al ritmo más oportuno y apropiado para su tarea (Harashim, 2000, p.25).

Se entiende la RVA como un tipo de comunidad virtual que surge de la red cuando un conjunto de personas llevan a cabo discusiones públicas que tienen al menos las siguientes características: son extensas y se presentan por tiempo prolongado, son regulares y denotan sentido humano en las relaciones personales que sostienen estas personas en el ciberespacio. Son grupos que surgen en la virtualidad, con el propósito de aprender, bien sea de manera formal o informal, mediante una práctica metodológica acordada o no.

Las RVA se orientan por metas comunes y experiencias compartidas, así como también por una membrecía, identidad de grupo, sentido de pertenencia, una zona del ciberespacio compartida y frecuentada por sus miembros, contacto social a través de la comunicación mediada por computador y sostenida en el tiempo, lo que garantiza la creación de lazos sociales y afectivos entre sus miembros (Sánchez, 2001). Esto tiende a mejorar las vías tradicionales de enseñanza y aprendizaje, abriendo nuevos caminos para la comunicación, la colaboración y la producción de conocimientos de una comunidad de discusión entre iguales, en donde la participación fluye dependiendo del interés de los participantes por intercambiar información y conocimiento.

En el campo educativo, el propósito de las redes virtuales de aprendizaje (Eisemberg y Ely, citados por Harasim, 2000) ha sido expandir las posibilidades de la enseñanza y del aprendizaje, desde el aumento en la calidad de la interacción, la motivación y el entusiasmo que presentan los estudiantes, su participación activa e independiente, la producción de textos individuales y colectivos, las posibilidades de interacción entre pares o co- 
legas, de aprendizaje colaborativo y la posibilidad de establecer una comunicación cercana entre los miembros del grupo.

A nivel educativo se han caracterizado tres tipos de RVA (Harasim, 2000): 1) modo adjunto, en donde se busca facilitar una opción de comunicación entre estudiantes, docentes y la comunidad escolar, fuera del aula presencial y del horario establecido, ampliando la discusión y los debates planteados en la clase, para lograr un mayor acceso a los profesores, intercambiar ejercicios de la clase, facilitar el trabajo en grupo por parte de los estudiantes y ampliar las oportunidades de interacción social; 2) modo mixto, cuando se convierte en el medio de desarrollo de algunas temáticas de un curso, integradas en el programa académico mediante actividades como la realización de juegos de simulación, el estudio de casos y la revisión de trabajos realizados en parejas o grupos pequeños de participantes; 3) modo de red, en donde todo un curso se realiza en entornos informáticos como forma principal de discusión e interacción académica entre los participantes y el docente, con el objetivo de cumplir objetivos de aprendizaje por parte de los estudiantes.

Estos tipos de RVA representan los tipos de uso de las TIC que se emplean para recrear o generar entornos de aprendizaje o espacios de trabajo específicos, que existen, esencialmente gracias a ellas, que pueden ser espacios de trabajo o entornos de aprendizaje individuales o colaborativos y puede tratarse de espacios o entornos que operen en paralelo o simultáneamente (Coll, Mauri y Onrubia, 2008).

En estos tipos de RVA, el entorno virtual se convierte en un complemento del desarrollo de una temática de trabajo, en un espacio comunicativo y colaborativo de discusión o en el entorno principal de interacción (Sánchez, 2001). En cualquiera de estos casos, es necesario que se establezcan relaciones claras con el proceso de aprendizaje de los participantes por lo cual las intenciones pedagógicas y comunicativas deben ser objeto del proceso de diseño de las actividades y la dinámica de la RVA.
Desde este punto de vista, la RVA puede entenderse como un mediador de los procesos de aprendizaje pues, a pesar de que el aprendizaje es un proceso individual, no colectivo, está influido por una variedad de factores externos, incluyendo las interacciones grupales e interpersonales, que implican el uso del lenguaje en la reorganización y modificación de las estructuras de conocimiento y comprensión de cada persona, por lo que el aprendizaje es simultáneamente un fenómeno social y privado (Kaye, 1991), que puede ser favorecido ampliamente por la RVA.

En este punto, se puede establecer un contraste entre las investigaciones consultadas sobre conocimiento escolar y RVA en cuanto al papel del docente y las formas de registro del proceso de construcción de conocimiento. Mientras que en las primeras, la construcción de conocimiento escolar está ligada al conocimiento profesional del profesor $y$, al parecer, la única manera de evidenciarla es mediante la realización de estudio de caso de su actividad en el aula de clase, en las investigaciones sobre RVA, el rol del docente se transforma y aparecen nuevos medios de evidenciar los procesos de construcción, lo que permite suponer que la construcción de conocimiento escolar, apoyada en una RVA, podría tener características particulares.

\section{¿Cómo avanza la reflexión?}

En la educación apoyada en medios virtuales el profesor deja de ser el "poseedor" del conocimiento y gestor del proceso de enseñanza aprendizaje, para convertirse en un "par académico", en otro mediador de conocimiento y en el diseñador del ambiente de aprendizaje en tanto que es él quien define los enfoques, estrategias y actividades que posibilitan los aprendizajes de los estudiantes. En esta medida, el docente debe ser un conocedor no solo de los contenidos del área, sino también de las estrategias pedagógicas virtuales y del manejo.

En los procesos construcción de conocimiento escolar, por parte de los estudiantes, la presencia del docente en la RVA puede convertirse en una variable que interviene con gran influencia en los procesos de interacción de los estudiantes. 


\section{FIGURA 3. Perspectiva didáctica de la RVA}

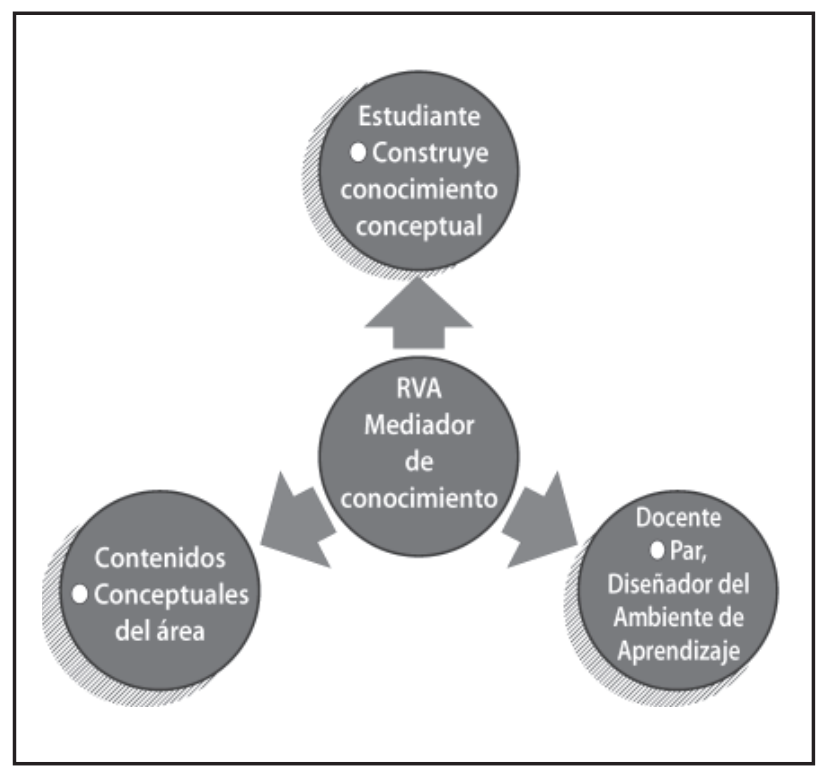

Fuente: elaboración propia

El proceso de diseño de una RVA se torna, en este punto, como un elemento esencial para su desarrollo. En este aspecto, se han encontrado dos proyectos de investigación que se toman como referentes para la construcción de ambientes virtuales de aprendizaje. El primero de ellos es una experiencia de investigación desarrollada por la Red Universitaria José Celestino Mutis (Unigarro, 2003) que se orienta a la conformación de comunidades virtuales de aprendizaje entre docentes.

Este proyecto busca caracterizar las relaciones que se presentan entre el proceso de formación apoyado en medios virtuales de ciento veinte docentes de las universidades que conforman la Red, en el tema de producción de contenidos virtuales y la conformación de una comunidad virtual de aprendizaje.

Utilizando una metodología de carácter cualitativo, fundamentada en el estudio de caso, se recolecta información generada por los docentes en el curso de formación y la producción de contenidos virtuales diseñados y desarrollados colaborativamente, en diversas áreas de conocimiento a nivel de formación profesional. Este proyecto plantea que los contenidos son diseñados y desarrollados, por parte de los profesores, a partir de cuatro ele- mentos: modelo pedagógico, comunicativo, técnico y de contenidos (Unigarro, et al, 2003).

En las conclusiones del proyecto se menciona que el mayor obstáculo para lograr el diseño y desarrollo de los cursos prototipo fue el tiempo, tanto de disponibilidad de los participantes como de desarrollo de las tareas, dadas la necesidad de asincronía en los procesos de interacción, pues se requiere de la construcción de un contexto académico compartido que favorezca la constitución de un colectivo de personas con un proyecto común a alcanzar, a partir de la resignificación y redimensionamiento de la enseñanza de las disciplinas desde la construcción colaborativa, las especialidades de cada uno de los participantes y el reconocimiento y aceptación del otro como par académico, sin embargo se encontraron pocas evidencias del desarrollo de un trabajo colaborativo en este sentido (Unigarro, 2003).

Este modelo de diseño es retomado por Molina y Briceño (2006) en el proceso de conformación de una RVA y se caracterizan los componentes del diseño de carácter pedagógico, que orienta el tipo de aprendizaje que se apoya en la red, las actividades, contenidos y el tipo de relaciones entre los actores educativos; a nivel técnico, desde la arquitectura tecnológica de la red, los módulos y mapas de navegación en relación con la estructura de la red, las plataformas y programas a utilizar; la comunicación que pretende establecer entre los participantes de la red, los niveles de interactividad y su correspondencia con el diseño de interfaz; y a nivel administrativo, que determina el tipo de roles de los participantes, la administración de contenidos y protocolos de registro.

Es necesario que en esta reflexión sobre la construcción de conocimiento escolar y el diseño de contenidos se conviertan en un aspecto central, dado que en la RVA se espera albergar materiales de apoyo y actividades de aprendizaje, que a partir de la interacción permitan caracterizar el proceso de construcción conceptual.

Los elementos mencionados en este artículo llevan a colegir que las RVA pueden, efectivamente, ser 
un elemento mediador en los procesos de construcción de conocimiento en el contexto escolar, y con las características epistemológicas de este tipo de conocimiento y atendiendo a sus dinámicas evolutivas.

Luego de este recorrido por algunos de los antecedentes de investigación en el tema, pueden surgir muchas dudas, preguntas e inquietudes que pueden orientar nuevos procesos investigativos, que muestren opciones y posibilidades para que los docentes puedan orientar los procesos de enseñanza y aprendizaje de sus estudiantes. Este es entonces, un camino que apenas inicia.

\section{Bibliografía}

Angulo, F.; Garzón, D.; Pabón O.; Mejía, M. F.; Obando, G. y Posada, F. (2007). Aspectos Teóricos y Metodológicos para la Consolidación de una Red de Aprendizaje desde la Didáctica de las Matemáticas. Colciencias, Universidad del Valle, Universidad de Antioquia.

Beirute, L. y Barahorna, J. C. (2004). Los mapas conceptuales en el contexto de las redes sociales: un nuevo escenario de aplicación. En: Cañas, A. J.; Novak, J. D. y González, F. M. (Eds). First Int. Conference on Concept Mapping. Pamplona.

Bonilla, M.; Molina, R.; Martínez, L. y Narváez, D. (2007). Red virtual de aprendizaje del área de matemáticas, como estrategia para la formación e investigación docente. Informe final de investigación: Colciencias-MEN-Universidad Distrital Francisco José de Caldas.

Burgos, S. D. (2006). Estudio de la estructura y del comportamiento de las comunidades virtuales de aprendizaje no formal sobre estandarización del e-learning. (Tesis doctoral). Universidad Europea de Madrid.

Coll, C.; Mauri, M. T. y Onrubia, J. (2008). “Análisis de los usos reales de las Tic en los contextos educativos formales: una aproxima- ción sociocultural”. En: Revista Electrónica de Investigación Educativa. 10 (1).

Di Franco, G.; Di Franco, N. y Siderac, S. (2004). "El curriculum enseñado y el lugar del conocimiento". En: Anuario de la Facultad de Ciencias Humanas. Universidad de la Pampa. (6): 175-187

Engel, R. A. (2008). Construcción de conocimiento en entornos virtuales de enseñanza y aprendizaje. (Tesis doctoral). Universidad de Barcelona. Acceso: Agosto de 2009. Disponible en: http://www.tesisenxarxa.net/TDX-0123109. $115623 /$

Gross, B. (s,f.). El aprendizaje colaborativo a través de la red: límites y posibilidades. Acceso: enero de 2009. Disponible en: http://www.uninorte. edu.co/congresog10/conf/08_El_Aprendizaje_Colaborativo_a_traves_de_la_red.pdf.

Johnson, D. W. \& Roger, T. J. (1998). "Cooperative Learning, Values, and Culturally Plural Classrooms". Cooperative Learning Center at the University of Minnesota. Minneapolis. Access: mayo de 2007. In: http://www.clcrc. com/pages/CLandD.html.

Kaye, A. (Ed.) (1991). Collaborative Learning Through Computer Conferencing. The Najaden Papers. NATO ASI Series. Springer-Verlag Berlin.

Maldonado, L. F., Serrano, E. (2008). "Construcción de una red de aprendizaje". En: Revista Nómadas. 29 (Abril): 211-222

Martinez R. C. A. (2006). "Las propuestas de conocimiento escolar en los inicios del aprendizaje de la química: un estudio de caso en las clases de química”. En: Revista Tumbaga. (1): 129-144.

Molina, R. y Briceño, S. (2006). Conformación de redes virtuales de aprendizaje entre maestros de educación media y básica. Informe final de investigación: Universidad Distrital Francisco José de Caldas. 
Ochoa, A. y Petrizzo, M. (s.f.). "Redes cognitivas y redes de aprendizaje: hacia la construcción de conocimiento crítico en red". En: REDES Revista

Hispana para el análisis de redes sociales. Acceso: agosto de 2009. Disponible en: http://revistaredes.rediris.es.

Ontalba, y R. J. A. (2002). “Comunidades virtuales académicas y científicas españolas”. En: Profesional de la información. 11 (5): 328-338.

Osorio, L. A. y Sánchez, A. (2000). "Ambientes colaborativos en ludomática”. En: Revista de Informática Educativa. 13 (1): 41-57.

Sánchez, J. (2001). Comunidades virtuales de aprendizaje: conceptos e ideas. Acceso: junio de 2008. Disponible en: http://www.dcc.uchile.cl.
Tobon, M. I. (2001). "Acción comunicativa y educación virtual". En: Revista de Ciencias Humanas. UTP. (32):151-162.

Unigarro, M.; Castaño, L. A.; Mestre, G.; Molina R.; Prado, M.; Ruiz, E. y Victoria, N. (2003) Las comunidades virtuales de aprendizaje en el contexto de las redes universitarias. Aceso: enero de 2009. Disponible en: http://sev.cuao. edu.co/pages/docs/comuvirt_aprendizaje_ universitarias.pdf.

Vásquez, B. B.; Jiménez, P. R.; Mellado, J. V. y Taboada, L. C. (2006). "El análisis de la epistemología del conocimiento escolar". En: Revista Mexicana de Investigación Educativa. 11 (31): 1259-1286

Vygostki, L. (1934). Pensamiento y lenguaje. Argentina: Buenos Aires Editorial La Pléyade. 